\title{
A case of primary hyperparathyroidism due to an intrathymic ectopic parathyroid adenoma in a 15-year-old boy
}

Youngseok Seo, MD,

Kyungchul Song, MD,

Han Saem Choi, MD,

Junghwan Suh, MD,

Ahreum Kwon, MD,

Hyun Wook Chae, MD, PhD,

Ho-Seong Kim, MD, PhD

Department of Pediatrics, Severance Children's Hospital, Endocrine Research Institute, Yonsei University College of Medicine, Seoul, Korea
Received: 1 November, 2019

Revised: 4 December, 2019

Accepted: 30 December, 2019

Address for correspondence: Ho-Seong Kim, MD, PhD

Department of Pediatrics, Severance Children's Hospital, Endocrine Research Institute, Yonsei University College of Medicine, 50-1 Yonsei-ro, Seodaemun-gu, Seoul 03722, Korea Tel: +82-2-2228-2069

Fax: +82-2-393-9118

E-mail:kimho@yuhs.ac

https://orcid.org/0000-0003-1135099X
Hypercalcemia due to primary hyperparathyroidism (PHPT) is uncommon in children. PHPT is typically caused by a single parathyroid adenoma. Ectopic parathyroid adenomas account for $6 \%-16 \%$ of all parathyroid adenomas and are rare in children but should be considered in cases that present with hypercalcemia. We report the case of a 15-year-old boy with PHPT due to an intrathymic ectopic parathyroid adenoma. Neck ultrasonography and Tc-99m-sestamibi (MIBI) scanning with single-photon emission computed tomography/computed tomography (SPECT/CT) revealed ectopic parathyroid adenoma in the thymus. Video-assisted thoracoscopic surgery was performed to remove the ectopic parathyroid adenoma. Pathology showed intrathymic ectopic parathyroid adenoma. After surgery, the patient's serum calcium level immediately normalized. Intact parathyroid hormone (iPTH) and alkaline phosphatase levels returned to normal ranges within 3 months. Delayed diagnosis of PHPT can cause end-organ damage; a timely diagnosis is especially critical to preserve bone and renal function. If ectopic parathyroid adenomas are well localized in preoperative imaging evaluation and intraoperative iPTH level decreases after resection, ectopic parathyroidectomy without bilateral neck exploration may be performed to avoid unnecessary morbidity.

Keywords: Primary hyperparathyroidism, Hypercalcemia, Ectopic parathyroid gland, Parathyroid adenoma, Child

\section{Introduction}

Hypercalcemia is uncommon in children; patients typically present with characteristic signs/symptoms or may be diagnosed incidentally following investigations for a variety of nonspecific conditions. ${ }^{1)}$ The causes of hypercalcemia in children can be classified as parathyroid hormone (PTH)-dependent or PTH-independent and may be either congenital or acquired. ${ }^{2)}$ Primary hyperparathyroidism (PHPT) accounts for $>90 \%$ of hypercalcemia in adults, and most patients with PHPT present with a single adenoma (80\%) or hyperplasia $(15 \%-20 \%)$ of the parathyroid gland. Parathyroid carcinoma occurs rarely, accounting for $<1 \%$ of all cases of PHPT. ${ }^{3)}$ In contrast, PHPT cases are rare in children and are likely to account for $<5 \%$ of the cases of hypercalcemia in children. ${ }^{2)}$ Pediatric PHPT may occur due to genetic causes, such as familial hypocalciuric hypercalcemia $(\mathrm{FHH})$, familial isolated hyperparathyroidism, and multiple endocrine neoplasia (MEN). ${ }^{2,4)}$ PHPT is caused by a solitary parathyroid adenoma in $80 \%$ of diagnosed adults ${ }^{5)}$ and $65 \%-70 \%$ of children. ${ }^{6.7)}$ In a previous study, the authors reported that ectopic parathyroid adenoma accounts for 6\%-16\% of total parathyroid adenomas, and the most common ectopic location is the thymus, which accounts for $38 \%$ of ectopic parathyroid adenomas. ${ }^{8)}$ In all pediatric PHPT patients, surgical resection of the parathyroid gland is required for definitive treatment. ${ }^{3)}$ After curative 
parathyroid resection, the prognosis is usually favorable without complications. ${ }^{9)}$

Here, we report a rare case of PHPT due to ectopic parathyroid adenoma in the thymus in a 15-year-old boy along with a comprehensive literature review about the case.

\section{Case report}

A 15-year-old male patient was born at 29 weeks of gestation with a birth weight of $1.12 \mathrm{~kg}$. At 3 years of age, he was diagnosed with cerebral palsy, spastic diplegia, and developmental delay. He had a history of orthopedic surgery in 2009 for progression of a foot deformity due to spasticity of the lower limb and gait disturbance. The patient was able to walk independently with the aid of a walker. However, due to the discomfort that resulted from walking with a crouching gait, a distal femoral extension and shortening osteotomy were planned. The initial preoperative laboratory evaluation indicated hypercalcemia and elevated alkaline phosphatase (ALP), so he was referred to the Department of Pediatric Endocrinology.

The patient had no family history of endocrinologic disease or tumor. History taking revealed no previous fracture or any medico-surgical history except as described above. Although he had reported more discomfort when walking one year prior, there were no specific hypercalcemia symptoms, such as anorexia, weight loss, or mood changes. He showed no signs or symptoms of nephrolithiasis. Upon physical examination, his height was $159 \mathrm{~cm}$, which was assigned to the $3 \mathrm{rd}-5$ th percentile; his yearly growth rate was in the normal range of about $6 \mathrm{~cm}$, and his weight was $56.9 \mathrm{~kg}$, which was in the 25th50th percentile. There had been an increase in existing right genu valgum compared to that from the radiograph from the prior year (Fig. 1). No mass was palpable on the neck.

Laboratory evaluation showed hypercalcemia $(12.8 \mathrm{mg} /$ $\mathrm{dL}$; reference range, $8.5-10.5 \mathrm{mg} / \mathrm{dL})$, hypophosphatemia (2.0 $\mathrm{mg} / \mathrm{dL}$; reference range, $3.8-5.9 \mathrm{mg} / \mathrm{dL}$ ), ALP > 2,300 IU/L (reference range, 81-316 IU/L), elevated intact PTH (iPTH, $1,930 \mathrm{pg} / \mathrm{mL}$; reference range, $15-65 \mathrm{pg} / \mathrm{mL}$ ), vitamin $\mathrm{D}$ deficiency (25-OH-vitamin D level of $6.09 \mathrm{ng} / \mathrm{mL}$; reference range, 30-100 $\mathrm{ng} / \mathrm{mL})$, and elevated $1 \mathrm{a}-25(\mathrm{OH})$-vitamin D level (115.9 pg/mL; reference range, $19.6-54.3 \mathrm{pg} / \mathrm{mL}$ ), which suggested PHPT rather than secondary or tertiary hyperparathyroidism. A 24-hour urinalysis revealed values suggestive of hypercalciuria (a urine-Ca-to-Cr ratio of 0.45 [29.8 mg/dL to $66.3 \mathrm{mg} / \mathrm{dL}]$ ). Fractional excretion of calcium was $1.3 \%$, which favors diagnosis of PHPT rather than FHH. To rule out genetic forms of PHPT, next-generation sequencing was performed using the Illumina Nextseq550 Sequence System (Illumina, Inc., San Diego, CA, USA), which showed no significant results corresponding with the clinical findings of MEN1, RET, HRPT2, or CASR.

Lower-extremity computed tomography (CT) revealed multiple, variable-sized osteolytic lesions in the bilateral iliac bones, femurs, and tibia. Radiographs of the hands and skull also showed low bone density (Fig. 2). Dual-energy x-ray absorptiometry was performed, and bone mineral density (BMD) of the lumbar spine (L1-4) was $0.626 \mathrm{~g} / \mathrm{cm} 2$ with a Z-score of -2.3, which is suggestive of low BMD. Renal ultrasonography showed mild medullary nephrocalcinosis without stones or hydronephrosis.

To evaluate PHPT, neck ultrasonography and Tc-99msestamibi (MIBI) scanning with single-photon emission computed tomography/CT (SPECT/CT) were carried out. Neck ultrasonography revealed no abnormal lesions except a

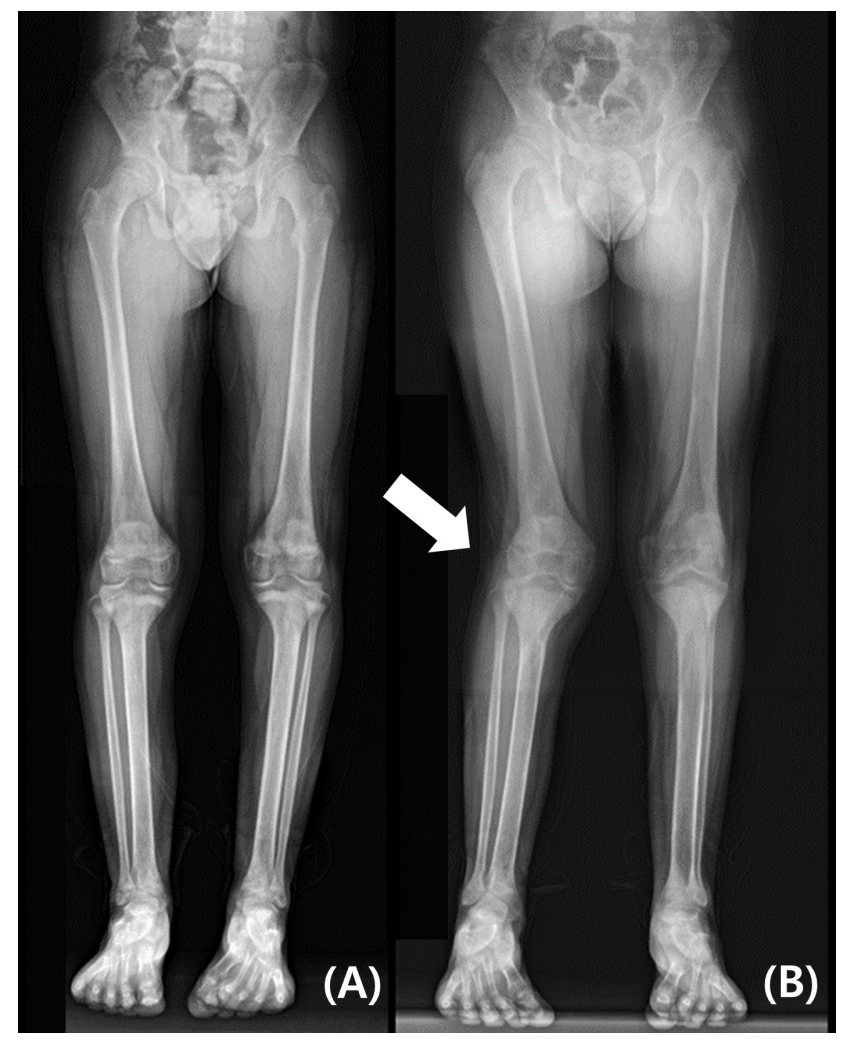

Fig. 1. Long-bone radiographs were recorded yearly. This 1-year follow-up radiograph (B) shows increased right genu valgum (white arrow) compared to that of the previous year (A).

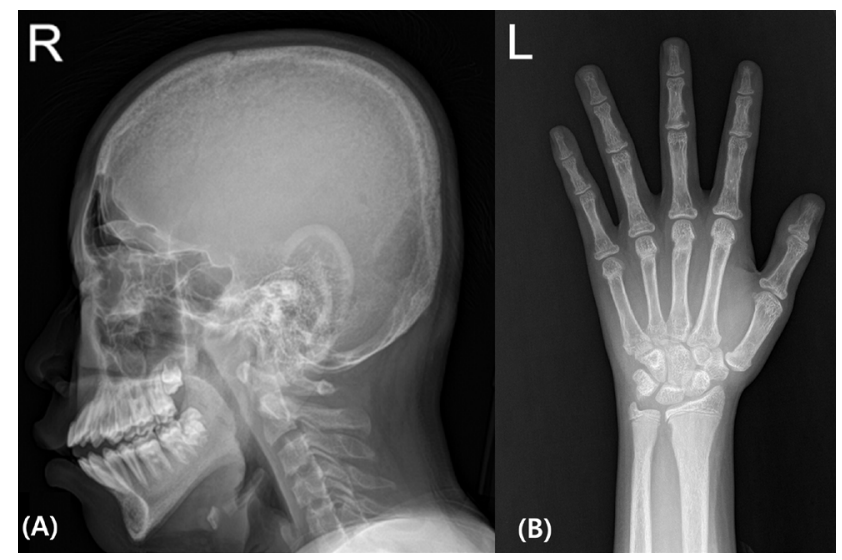

Fig. 2. Skull (A) and hand radiographs (B) indicate low bone density. 


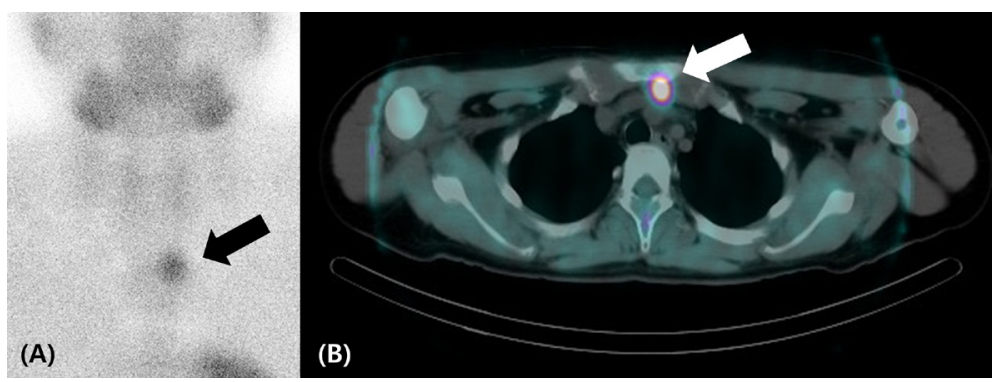

Fig. 3. An ectopic parathyroid adenoma is detected in the prevascular region by $99 \mathrm{mTc}$-sestamibi scanning ( $A$, black arrow) and single-photon emission computed tomography/computed tomography (B, white arrow).

Table 1. Pre- and postoperative biochemical laboratory studies in ectopic parathyroid adenoma

\begin{tabular}{lccccc}
\hline POD & $\begin{array}{c}\text { Calcium } \\
(8.5-10.5 \mathrm{mg} / \mathrm{dL})\end{array}$ & $\begin{array}{c}\text { Phosphate } \\
(3.8-5.9 \mathrm{mg} / \mathrm{dL})\end{array}$ & $\begin{array}{c}\text { ALP } \\
(81-316 / \mathrm{U} / \mathrm{L})\end{array}$ & $\begin{array}{c}\text { iPTH } \\
(15-65 \mathrm{pg} / \mathrm{mL})\end{array}$ & $\begin{array}{c}25-\mathrm{OH}-\mathrm{vitamin} \mathrm{D} \\
(30-100 \mathrm{ng} / \mathrm{mL})\end{array}$ \\
\hline Preoperative & 12.8 & 2.0 & $>2300$ & 1930.0 & 6.09 \\
POD 0 & 7.7 & 2.3 & $>2300$ & 36.5 & - \\
POD 12 & 8.9 & 3.6 & 2193 & 117.0 & 20.5 \\
POD 96 & 9.9 & 5.3 & 436 & 41.6 & 34.44 \\
\hline
\end{tabular}

POD, postoperative day; ALP, alkaline phosphatase; iPTH, intact parathyroid hormone.

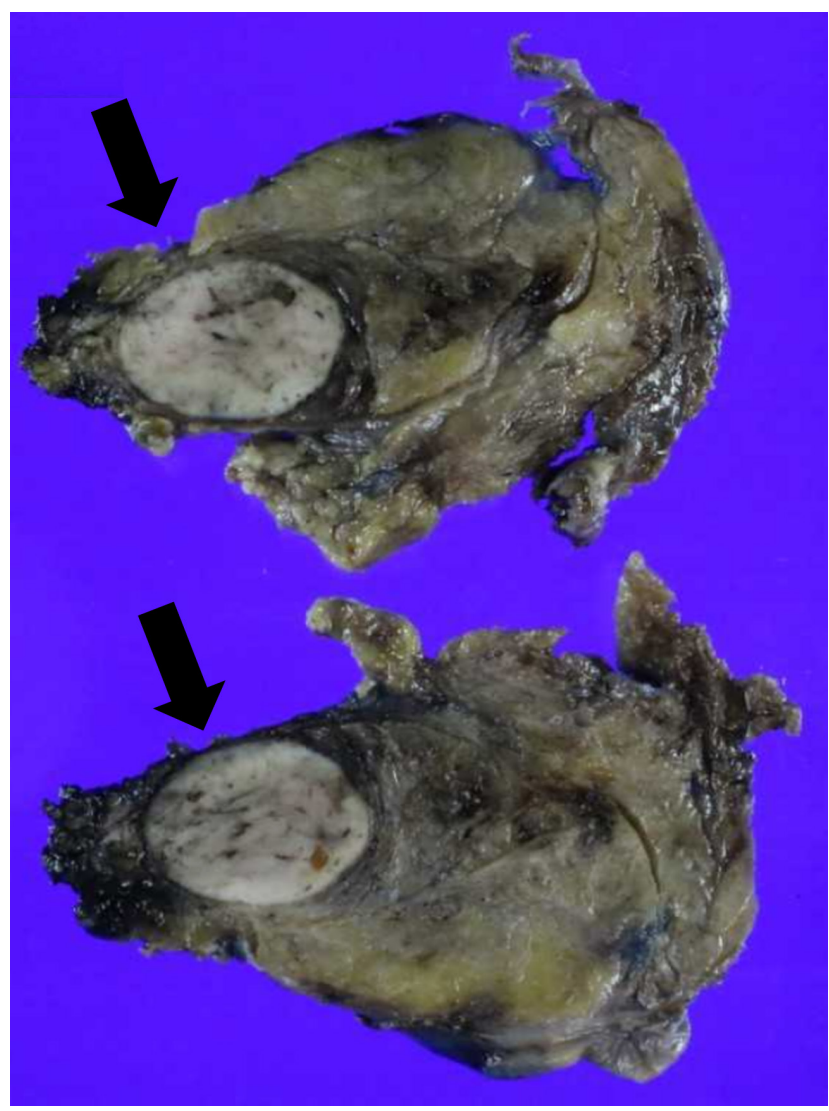

Fig. 4. The gross appearance of an ectopic parathyroid adenoma. A welldemarcated mass $2.9 \mathrm{~cm} \times 1.5 \mathrm{~cm}$ (black arrow) in size located within the thymus.

tiny colloid cyst in the thyroid gland. The MIBI scan showed a round-shaped nodular lesion suggestive of ectopic parathyroid adenoma in the prevascular region (Fig. 3).

Oral calcidiol was started before surgery. After localizing the ectopic parathyroid adenoma, we consulted with endocrine surgeons and thoracic surgeons and finally decided to perform video-assisted thoracoscopic surgery. Intraoperative findings revealed a mass on the left side of the thymus. Thymectomy including the mass was performed. Intraoperative iPTH (ioPTH) level decreased from $1,584 \mathrm{pg} / \mathrm{mL}$ to $36.5 \mathrm{pg} / \mathrm{mL}$, which is within the normal range. Sectioning of the specimen was performed, and it showed a whitish to tan-colored, welldemarcated mass $(2.9 \mathrm{~cm} \times 1.5 \mathrm{~cm})$ within the thymus (Fig. 4). Pathological findings suggested an intrathymic ectopic parathyroid adenoma.

After surgery, serum calcium dropped sharply from 12.8 $\mathrm{mg} / \mathrm{dL}$ to $7.7 \mathrm{mg} / \mathrm{dL}$. The oral calcidiol given before surgery was discontinued in favor of another calcium carbonatecalcidiol combination oral agent to provide more calcium and calcidiol after surgery. On postoperative day 1 , serum calcium level increased from $7.7 \mathrm{mg} / \mathrm{dL}$ to $8.1 \mathrm{mg} / \mathrm{dL}$. The patient showed no signs or symptoms of hypocalcemia, so he was discharged on postoperative day 1 with a prescription for an oral agent containing elemental calcium $26.4 \mathrm{mg} / \mathrm{kg} /$ day and cholecalciferol 2,400 IU/day. He was followed up on postoperative day 12 at an outpatient clinic. He showed normalized levels of calcium $(8.9 \mathrm{mg} / \mathrm{dL})$ and phosphate $(3.6$ $\mathrm{mg} / \mathrm{dL}$ ). His ALP level had slightly decreased from $>2,300 \mathrm{IU} /$ $\mathrm{L}$ to $2,193 \mathrm{IU} / \mathrm{L}$. The iPTH level increased from $36.5 \mathrm{pg} / \mathrm{mL}$ (immediately postoperatively) to $117.0 \mathrm{pg} / \mathrm{mL}$. The $25-\mathrm{OH}-$ vitamin D level increased from $6.09 \mathrm{ng} / \mathrm{mL}$ to $20.05 \mathrm{ng} / \mathrm{mL}$. Three months after surgery, the ALP level had decreased from 2,193 IU/L to $436 \mathrm{IU} / \mathrm{L}$, and the iPTH level fell from $117.0 \mathrm{pg} /$ 
$\mathrm{mL}$ to $41.6 \mathrm{pg} / \mathrm{mL}$, which was within the normal range (Table 1 ).

\section{Discussion}

Hyperparathyroidism is very rare in the pediatric population, with an estimated prevalence of 2-5 cases per 100,000 people); PHPT due to ectopic parathyroid adenoma is extremely rare, with only a few published case reports. ${ }^{10)}$ The most common ectopic site for a parathyroid gland is the thymus. ${ }^{8)}$ In a study analyzing 202 patients with ectopic parathyroid gland, the ectopic parathyroid sites were most common in the thymus (38\%), followed by the retroesophageal (31\%) and intrathyroidal regions $(18 \%)$. In our case, ectopic parathyroid adenoma was identified in the thymus, suggesting that this region should be evaluated when attempting to locate ectopic parathyroid adenomas in children.

Unlike adult patients, almost $80 \%$ of pediatric patients with PHPT were symptomatic with target organ involvement. ${ }^{6,9}$ However, signs and symptoms of PHPT may not be obvious in the early stages; therefore, diagnosis requires careful observation. In our case, the patient did not show specific signs or symptoms except increased genu valgum. As genu valgum is a rare skeletal manifestation in children, ${ }^{11)}$ PHPT is not immediately suspected in the presence of this manifestation. Children with PHPT can present with nonspecific symptoms, such as fatigue, poor appetite, weight loss, headache, polyuria, polydipsia, nausea, emesis, abdominal pain, diarrhea, depression, or joint pain. ${ }^{6}$ These vague clinical manifestations of PHPT lead to delayed diagnosis that allows end-organ damage; a timely diagnosis is especially critical to preserve bone and renal function. ${ }^{9}$ Therefore, if children have persistent, nonspecific symptoms that cannot be attributed to other disease processes, screening laboratory studies to assess the serum levels of calcium and iPTH should be performed. ${ }^{6}$

Surgical resection of the parathyroid glands in PHPT provides a definitive cure. In adult populations, surgery is recommended if the patient meets any of the following criteria: hypercalcemia that is consistently $>1 \mathrm{mg} / \mathrm{dL}$ above normal; fracture; nephrocalcinosis, hypercalciuria, and other risk factors for renal stones; reduced BMD (T-score $<-2.5$ at any site); and age $<50$ years. ${ }^{3,12)}$ Therefore, all patients with PHPT in the pediatric age group require surgical resection for definitive treatment. ${ }^{13)}$ Accordingly, preoperative parathyroid imaging to localize an ectopic parathyroid is necessary in children who are scheduled to undergo surgery. In a meta-analysis of 43 preoperative localization technique studies, ultrasonography had a pooled sensitivity of $76.1 \%$ and a positive predictive value of $93.2 \%$, while MIBI-SPECT had a sensitivity of $78.9 \%$ and a positive predictive value of $90.7 \%{ }^{14)}$ In our case, combined ultrasonography and MIBI scanning with SPECT/CT successfully detected ectopic intrathymic parathyroid adenoma.

Most adult patients with PHPT have abnormal parathyroid lesions in the neck, and resection of any abnormal parathyroid glands can be performed through a standard cervical approach.
Even in cases with abnormal parathyroid lesions that extend into the mediastinum, most can be removed through a cervical approach. Only $2 \%$ of all PHPT require direct mediastinal exploration. ${ }^{15,16)}$ Traditionally, a transsternal or transthoracic approach involving an open technique has been used in cases with deep mediastinal parathyroid lesions. ${ }^{15)}$ Recently, minimally invasive parathyroidectomy for PHPT has been adopted at many centers to avoid unnecessary morbidity. ${ }^{12}$ Recent studies have shown that minimally invasive mediastinal ectopic parathyroidectomy is safe and feasible if the anatomical position of any lesions is well determined and ioPTH monitoring is available. ${ }^{17,18)}$ However, there are no specific guidelines for management of ectopic parathyroid adenoma in children. Surgical treatment for PHPT in children should be individualized according to nature and location of the abnormal parathyroid glands. ${ }^{6,90)}$ In our case, the patient had no family history of endocrine tumors, and the laboratory findings did not suggest MEN-I, MEN-IIa, or FHH. Therefore, surgery was performed before the genetic results were obtained. However, genetic testing should be carried out in PHPT patients who meet the following criteria: young age ( $<45$ years of age); multigland disease; carcinoma or atypical adenoma; a firstdegree relative who is a known mutation carrier; an index case with 2 or more MEN syndrome-associated tumors. ${ }^{2}$ In cases where MEN affects multiple glands, subtotal or total parathyroidectomy is recommended. ${ }^{19)}$ If ectopic parathyroid adenoma is well localized and ioPTH level decreases sharply after resection, minimally invasive parathyroidectomy without bilateral neck exploration could be a better option to avoid unnecessary morbidity in children.

\section{Ethical statement}

This study was approved by the Institutional Review Board of Yonsei University Hospital (4-2019-0907). Informed consent was obtained from the participant's parents.

\section{Conflict of interest}

No potential conflict of interest relevant to this article was reported.

\section{References}

1. McNeilly JD, Boal R, Shaikh MG, Ahmed SF. Frequency and aetiology of hypercalcaemia. Arch Dis Child 2016;101:3447.

2. Stokes VJ, Nielsen MF, Hannan FM, Thakker RV. Hypercalcemic disorders in children. J Bone Miner Res 2017;32:2157-70.

3. Bilezikian JP. Primary hyperparathyroidism. J Clin Endocrinol Metabol 2018;103:3993-4004.

4. Cormier C. Genetic hypercalcemia. Joint Bone Spine 2019;86:459-66. 
5. Walker MD, Silverberg SJ. Primary hyperparathyroidism. Nat Rev Endocrinol 2018;14:115-25.

6. Kollars J, Zarroug AE, van Heerden J, Lteif A, Stavlo P, Suarez L, et al. Primary hyperparathyroidism in pediatric patients. Pediatrics 2005;115:974-80.

7. Nicholson KJ, McCoy KL, Witchel SF, Stang MT, Carty SE, Yip L. Comparative characteristics of primary hyperparathyroidism in pediatric and young adult patients. Surgery 2016;160:1008-16.

8. Roy M, Mazeh H, Chen H, Sippel RS. Incidence and localization of ectopic parathyroid adenomas in previously unexplored patients. World J Surg 2013;37:102-6.

9. Li CC, Yang C, Wang S, Zhang J, Kong XR, Ouyang J. A 10year retrospective study of primary hyperparathyroidism in children. Exp Clin Endocrinol Diabetes 2012;120:22933.

10. Liu X, Sun L, Shao M, Li P, Liu W, Zhang X, et al. Primary hyperparathyroidism due to ectopic parathyroid adenoma in an adolescent: a case report and review of the literature. Endocrine 2019;64:38-42.

11. Arambewela MH, Liyanarachchi KD, Somasundaram NP, Pallewatte AS, Punchihewa GL. Case report: rare skeletal manifestations in a child with primary hyperparathyroidism. BMC Endocr Disord 2017;17:45.

12. Udelsman R, Akerstrom G, Biagini C, Duh QY, Miccoli P, Niederle B, et al. The surgical management of asymptomatic primary hyperparathyroidism: proceedings of the Fourth International Workshop. J Clin Endocrinol Metab 2014;99:3595-606.
13. Khan AA, Hanley DA, Rizzoli R, Bollerslev J, Young JE, Rejnmark L, et al. Primary hyperparathyroidism: review and recommendations on evaluation, diagnosis, and management. A Canadian and international consensus. Osteoporos Int 2017;28:1-19.

14. Cheung K, Wang TS, Farrokhyar F, Roman SA, Sosa JA. A meta-analysis of preoperative localization techniques for patients with primary hyperparathyroidism. Ann Surg Oncol 2012;19:577-83.

15. Iihara M, Suzuki R, Kawamata A, Horiuchi K, Okamoto T. Thoracoscopic removal of mediastinal parathyroid lesions: selection of surgical approach and pitfalls of preoperative and intraoperative localization. World J Surg 2012;36:132734.

16. Kaplan EL, Yashiro T, Salti G. Primary hyperparathyroidism in the 1990s. Choice of surgical procedures for this disease. Ann Surg 1992;215:300-17.

17. Chou PL, Chao YK, Liu YH. Minimally invasive removal of mediastinal ectopic parathyroid glands: a single-center experience. Formos J Surg 2019;52:6-10.

18. Said SM, Cassivi SD, Allen MS, Deschamps C, Nichols FC 3rd, Shen KR, et al. Minimally invasive resection for mediastinal ectopic parathyroid glands. Ann Thorac Surg 2013;96:1229-33.

19. Thakker RV, Newey PJ, Walls GV, Bilezikian J, Dralle H, Ebeling PR, et al. Clinical practice guidelines for multiple endocrine neoplasia type 1 (MEN1). J Clin Endocrinol Metabol 2012;97:2990-3011. 